\title{
Sensitivity Analysis in Medium Voltage Distribution Systems
}

\author{
Saad Ouali ${ }^{\# 1}$, Abdeljabbar Cherkaoui ${ }^{2}$ \\ 1,2 Laboratory of Innovative Technologies (LTI), \\ National School of Applied Sciences, \\ Abdelmalek Essaadi University \\ Tangier Morocco
}

\begin{abstract}
This paper presents a sensitivity analysis of radial distribution systems, with a focus on medium voltage distribution systems in Morocco, The proposed analysis can offer an analytical tool able to quantify how the network conductors characteristics and how the variation in active and reactive power loads and distributed generation connected to a medium voltage distribution systems may affect the voltage plan of the network. Obviously, any change in a system input impact the system performance. However, some inputs may have more impacts whereas others inputs may have less or more influence impacts. Voltage plan of radial systems is a function of their conductors' characteristics and connected active/reactive power. The main aim of this paper is to compute a sensitivity coefficient to quantify the impact of active and reactive power on the voltage plan, this type of sensitivity information is useful for estimating the expected voltage changes, and may also be used for choosing the optimal placement of distributed generation, reactive compensation and voltage control actuator. The proposed analysis had been applied to a radial distribution of 16 bus, this application had lead to know how a distributed generation can affect the voltage plan, and how changing its connected point, may lead to increase or decrease its impacts on the network performances. Also a comparison with several conductors characteristics with real underground cable and overhead lines information is presented in this paper, such a comparison had proved that network reinforcement can also be a choice for voltage control and had also lead to know the most impacted in underground and overhead networks by distributed generations integration and the migration from a passive to an active mode.
\end{abstract}

Keywords - Sensitivity Analysis, Radial distribution system, Backward/forward sweep, Medium voltage system.

\section{INTRODUCTION}

Medium voltage distribution systems in Morocco are radial with large number of nodes, branches, and complex topology configurations that can be changed for maintenance activities, emergency operations or network configurations.

The Moroccan medium voltage distribution system is made of 3 phases, with neutral grounded in the HV/MV substation. Two nominal magnitudes voltages are used $20 \mathrm{KV}$ and $22 \mathrm{KV}$.

Three forms of medium voltage distribution system can be distinguished: $100 \%$ overhead lines, $100 \%$ underground cables or a combination of both.

The underground networks are made of 3 single-phase cables. The overhead networks are made of three bares conductors, the most used topology is the radial configuration

The penetration of distributed generators in Medium Voltage (MV) distribution network will challenge the future grid operation. More intelligent methods should be used for a better utilization of the distribution network, in order to maintain, or even to improve, the power-supply reliability and quality. Voltage rise is normally the main limiting factor to prevent the increase of distributed generation in distribution networks [1].

The development of a voltage management strategy is a challenging task due to the non-linear relationship between the network load and the grid voltage. In the present paper we present a simple analytical tool to quantify voltage sensitivity due to the injection of active and reactive power at one or more nodes of MV distribution networks.

Sensitivity analysis in electric systems is an important tool to quantify the impact of variations in parameters on the system performance. Voltage regulation, loss reduction, network expansion planning, optimal placement of reactive sources, and many other applications regarding both planning and operation of power systems in which it is necessary, or even simply useful, to predict the changes of voltage magnitude caused by variations in loads and generations.

The main aim of this paper is to develop an analytical tool to quantify node voltage variations due to injections of active and reactive powers at one or more nodes of radial distribution networks. And to mitigate how node distances from the origin, section and type of conductor, overhead line or underground cable influence the network sensitivity.

In what follows, we present the impact of distributed generation on network voltage in section II. Next in Section III we provide the theoretical foundation of the method used to obtain mathematical expressions that link voltages sensitivity to node active and reactive powers variation, in section IV we present a case study of the proposed method with the use of the IEEE 15-bus system information, in section $\mathrm{V}$ we discuss the results obtained from the case study, and the comparisons with the Moroccan Medium Voltage system, section VI concludes this paper. 
II. IMPACTS OF DISTRIBUTED GENERATION ON THE NETWORK VOLTAGE

Considering the radial system shown in Fig1.

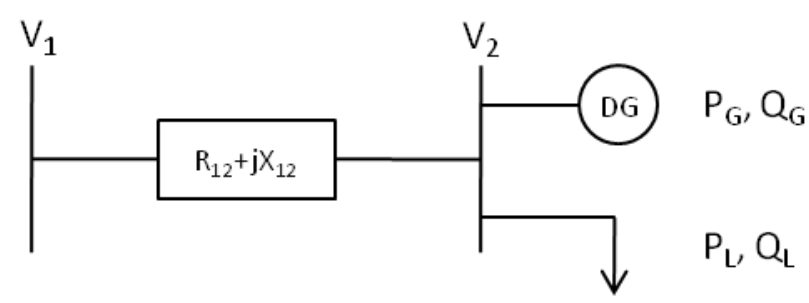

Fig 1: One line diagram to illustrate the voltage variation in a distribution system with DG.

In the case of any distributed generation (DG) connected to the node 2 , the relationship between the voltage of node 1 and 2 it:

$$
V_{1}-V_{2}=\frac{P_{12} r_{12}+Q_{12} x_{12}}{V_{2}}
$$

In the case of the presence of a distributed generation (DG) connect a the node 2 ,

$$
V_{1}-V_{2}=\frac{\left(P_{12}-P_{L}\right) r_{12}+/-\left(Q_{12}-Q_{L}\right) x_{12}}{V_{2}}
$$

Where $\mathrm{P}_{\mathrm{DG}}$ and $\mathrm{Q}_{\mathrm{DG}}$ are active and reactive power of the DG.

Equation (2) indicates that if the active power generated bu the DG is larger than the feeder load, power may flow from the DG to the substation and causes a voltage rise.

Equation (2) indicates too that, if the DG absorbs reactive power, the DG can either increase or decrease the voltage drop.

\section{PROPOSED SENSITIVITY ANALYSIS METHOD}

The most used analysis methods in electrical system: Gauss-Siedel method, Newton-Raphson method and Fast Decoupled method.

The first step is the construction of the Y-bus admittance matrix using the transmission line and transformer input data.

The Gauss-Seidel analysis method uses an iterative method based on Gauss equation, the Newton-Raphson is based on the expanding in Taylor's series about the initial estimate the active and reactive power formulation, the terms are limited to the first approximation of the equations. The Fast Decoupled Power analysis Method is one of the improved methods, which is based on a simplification of the Newton-Raphson method, the convergence is geometric.

A common procedure of the three methods, adopted for analysing electrical system in a power network is discussed in the pseudo-code shown in Fig.4 [8].

But medium voltage distribution systems in Morocco are characterized by a high $\mathrm{R} / \mathrm{X}$ rations and a strongly radial structure, which leads to ill-conditioned matrices and poor convergence characteristics of those load flow methods.

The problems have been revealed in a number of papers, where the classic transmission methods were not appropriate to solve practical problems presented when analysing distribution systems.
\# Start

\# Construction of the $Y_{\text {bus }}$ Matrix

\# Make an estimate of the voltage plan

\# Substitute the old values into power equations for the next iteration

\# Obtain the new value

\# compare the new value with Old value

\# If (New value - Old value) < specified tolerance; then end otherwise go to step 4.

Fig 2: Pseudo-code procedure for analyzing load flow in a power system

Several works had been presented in the scientific literature; the methods used can be divided into two principal categories: Methods based on the amelioration and adaptation of transmission methods such as fast-decoupled, and others are based on power summation method [3].

In what follow we adopt the power summation approach, so, let's consider [F] a column vectors, dimension ( $\mathrm{n} \times 1)$, whose elements are, the injections powers of network nodes.

$$
[F]=[V] *\left[I^{*}\right]=[P]+j[Q]
$$

Where $[\mathrm{P}]$ and $[\mathrm{Q}]$ are the column vectors, dimension $(\mathrm{n} \times 1)$ , whose elements are, respectively, the node active and reactive powers.

The complex conjugate of $[\mathrm{S}]$ is given by:

$$
\left[F^{*}\right]=\left[V^{*}\right] *[I]=[P]-j[Q]
$$

The current injections of network nodes are:

$$
[I]=\frac{[P]-j[Q]}{\left[V^{*}\right]}
$$

Current injections and relationship between the bus current injections and branch current can be obtained by applying Kirchhoff's current law (KCL) to the distribution network. The branch currents can then be formulated as functions of equivalent current injections.

A sample distribution system drawn below is taken on fig2 to illustrate the methodology chosen to develop a voltage sensitivity analysis method:

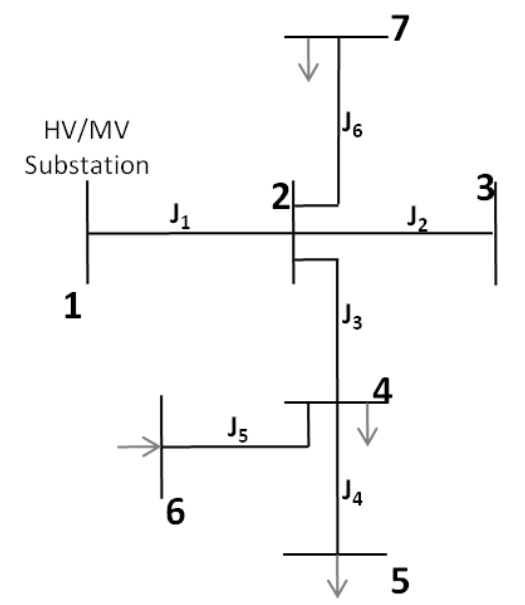

Fig 3: Single line diagram of a radial distribution network

By applying Kirchhoff's current law, the branch currents $\mathrm{I}_{1}, \mathrm{I}_{2}, \mathrm{I}_{3}, \mathrm{I}_{4}, \mathrm{I}_{5}, \mathrm{I}_{6}$ and $\mathrm{I}_{7}$ can be expressed by equivalent current injections as: 


$$
\begin{gathered}
J_{1}=I_{2}+I_{3}+I_{4}+I_{5}+I_{6}+I_{7} \\
J_{2}=I_{3} \\
J_{3}=I_{5}+I_{6} \\
J_{4}=I_{5} \\
J_{5}=I_{6} \\
J_{5}=I_{6} \\
J_{6}=I_{7}
\end{gathered}
$$

Therefore, the relationship between the bus current injections and branch currents can be expressed as:

$$
\left[\begin{array}{l}
J_{1} \\
J_{2} \\
J_{3} \\
J_{4} \\
J_{5} \\
J_{6}
\end{array}\right]=\left[\begin{array}{llllll}
1 & 1 & 1 & 1 & 1 & 1 \\
0 & 1 & 0 & 0 & 0 & 0 \\
0 & 0 & 1 & 1 & 1 & 0 \\
0 & 0 & 0 & 1 & 0 & 0 \\
0 & 0 & 0 & 0 & 1 & 0 \\
0 & 0 & 0 & 0 & 0 & 1
\end{array}\right]\left[\begin{array}{l}
I_{2} \\
I_{3} \\
I_{4} \\
I_{5} \\
I_{6} \\
I_{7}
\end{array}\right] \text { Or }[J]=[B I B C] \times[I]
$$

The constant BIBC matrix is an upper triangular matrix and contains values of 0 and +1 only.

The relationship between branch currents and bus voltages can be obtained as follows:

$$
\begin{aligned}
& V_{2}=V_{1}-J_{1} Z_{12} \\
& V_{3}=V_{2}-J_{2} Z_{23} \\
& V_{4}=V_{2}-J_{3} Z_{24} \\
& V_{5}=V_{4}-J_{4} Z_{45} \\
& V_{6}=V_{4}-J_{5} Z_{46} \\
& V_{7}=V_{2}-J_{6} Z_{27}
\end{aligned}
$$

Where $V_{i}$ is the voltage of bus $i$, and $Z_{i j}$ is the line impedance between node $\mathrm{i}$ and node $\mathrm{j}$. Substituting (8.1) and into (8.6), the equation (8.6) can be written as:

$$
V_{7}=V_{1}-J_{1} Z_{12}-J_{2} Z_{23}-J_{6} Z_{26}
$$

From (9), it can be seen that the bus voltage can be expressed as a function of branch currents, line parameters and the substation voltage. Similar procedures can be performed on other nodes; therefore the relationship between branch currents and bus voltages can be expressed as:

$$
\left[\begin{array}{l}
V_{1} \\
V_{1} \\
V_{1} \\
V_{1} \\
V_{1} \\
V_{1}
\end{array}\right]-\left[\begin{array}{l}
V_{2} \\
V_{3} \\
V_{4} \\
V_{5} \\
V_{6} \\
V_{7}
\end{array}\right]=\left[\begin{array}{llllll}
Z_{12} & 0 & 0 & 0 & 0 & 0 \\
Z_{12} & Z_{23} & 0 & 0 & 0 & 0 \\
Z_{12} & 0 & Z_{34} & 0 & 0 & 0 \\
Z_{12} & 0 & Z_{34} & Z_{45} & 0 & 0 \\
Z_{12} & 0 & Z_{34} & 0 & Z_{56} & 0 \\
Z_{12} & 0 & 0 & 0 & 0 & Z_{67}
\end{array}\right]\left[\begin{array}{l}
J_{1} \\
J_{2} \\
J_{3} \\
J_{4} \\
J_{5} \\
J_{6}
\end{array}\right]
$$

$$
\text { Or : } \quad[\Delta V]=[B C B V] \times[J]
$$

The BCBV matrix represents the relationship between branch current and bus voltages. The corresponding variations at bus voltage, generated by the variations at branch currents can be calculated directly by the BCBV matrix.

The BIBC and BCBV matrices are developed based on the topological structure of distribution systems. The BIBC matrix represents the relationship between bus current injections and branch currents. The corresponding variations at branch currents, generated by the variations at bus current injection can be calculated directly by the BIBC matrix. Combining equation (7) and (10.2), the relationship between bus current injections and bus voltages can be expressed as:

$$
[\Delta V]=[B C B V][B I B C][I]=[D L F][I]
$$

Where DLF is a multiplication matrix of BCBV and BIBC matrices

Let's consider two matrices $[\mathrm{R}]$ and $[\mathrm{X}]$ as :

$$
[\mathrm{R}]=\operatorname{real}([\mathrm{DLF}]) \text { and }[\mathrm{X}]=\mathrm{im}([\mathrm{DLF}])
$$

The element $(i, j)$ of the matrix $[R]$ is the sum of the resistance of the branches in which both $\mathrm{P}_{\mathrm{i}+1}$ and $\mathrm{P}_{\mathrm{j}+1}$ flow. For instance, in order to obtain the element $(3,2)$ of $[R]$, the branches in which both $\mathrm{P}_{4}$ and $\mathrm{P}_{3}$ flow are $\mathrm{J}_{1}$.

$$
[V]=\left[V_{1}\right]-([R]+j[X]) *\left(\frac{[P]-j[Q]}{\left[V^{*}\right]}\right)
$$

Simplified linear expressions can be derived from (13) under the following hypotheses (commonly accepted in distribution networks analysis) [4]:

- The phase difference between node voltages is negligible and, as a consequence, if phasor $V_{1}$ is chosen on the real axis, only the real part of voltage $[\mathrm{V}]=$ real $[\mathrm{V}]$ is considered.

- Constant current models are considered for loads (node powers are referred to system nominal voltage instead of actual node voltage).

Equations (13) can be written as:

For a node i:

$$
[V]=\left[V_{1}\right]-([R]+j[X]) *\left(\frac{[P]-j[Q]}{V_{\text {nom }}}\right)
$$

$$
V_{i}=V_{0}-\frac{1}{V_{\text {nom }}}\left(\sum R_{i j} P_{j}+\sum X_{i j} Q_{j}\right)
$$

From equation (15), the voltage of node $\mathrm{i}$ depend on the active and reactive power injections or consumed of all node networks.

As the node 1 is the slack bus, its voltage is always constant. The sensitivity $\left(S_{p}\right)_{i j}$ of voltage at node $\mathrm{i}$ with respect to the active power and reactive power at node $\mathrm{j}$, can be written as:

$$
\left\{\begin{array}{l}
\left(F_{p}\right)_{i j}=\frac{\partial V_{i}}{\partial P_{j}}=-\frac{1}{V_{n o m}} R_{i j} \\
\left(F_{q}\right)_{i j}=\frac{\partial V_{i}}{\partial Q_{j}}=-\frac{1}{V_{n o m}} X_{i j}
\end{array}\right.
$$

The total differential of function $\mathrm{V}_{\mathrm{i}}$ is:

$$
\begin{aligned}
d V_{i} & =\sum \frac{\partial V_{i}}{\partial P_{j}} d P_{j}+\sum \frac{\partial V_{i}}{\partial Q_{j}} d Q_{j} \\
& =\sum\left(S_{p}\right)_{i j} d P_{j}+\sum\left(S_{q}\right)_{i j} d Q_{j}
\end{aligned}
$$

Considering all network nodes

$$
\left[\begin{array}{c}
d V_{1} \\
\ldots \\
d V_{n}
\end{array}\right]=\left[\begin{array}{cccccc}
\frac{\partial V_{1}}{\partial P_{1}} & \cdots & \frac{\partial V_{n}}{\partial P_{1}} & \frac{\partial V_{1}}{\partial Q_{1}} & \ldots & \frac{\partial V_{n}}{\partial Q_{1}} \\
\ldots & \cdots & \ldots & \ldots & \cdots & \ldots \\
\frac{\partial V_{1}}{\partial P_{n}} & \cdots & \frac{\partial V_{n}}{\partial P_{n}} & \frac{\partial V_{1}}{\partial Q_{n}} & \ldots & \frac{\partial V_{n}}{\partial Q_{n}}
\end{array}\right] x\left[\begin{array}{c}
d P_{1} \\
\ldots \\
d P_{n} \\
d Q_{1} \\
\cdots \\
d Q_{n}
\end{array}\right]
$$


Let's consider [S], as the sensitivity matrix $(\mathrm{nx} 2 \mathrm{n})$, whose elements are the sensitivity of network nodes to active and reactive power variation.

The matrix $[\mathrm{F}]$, can be written as:

$$
\begin{gathered}
{[F]=\left[F_{p} \mid F_{q}\right]} \\
{\left[F_{p}\right]=\left[\begin{array}{ccc}
\frac{\partial V_{1}}{\partial P_{1}} & \cdots & \frac{\partial V_{n}}{\partial P_{1}} \\
\cdots & \cdots & \cdots \\
\frac{\partial V_{1}}{\partial P_{n}} & \cdots & \frac{\partial V_{n}}{\partial P_{n}}
\end{array}\right]=-\frac{1}{V_{n o m}}[R]} \\
{\left[F_{q}\right]=\left[\begin{array}{ccc}
\frac{\partial V_{1}}{\partial Q_{1}} & \cdots & \frac{\partial V_{n}}{\partial Q_{1}} \\
\cdots & \cdots & \cdots \\
\frac{\partial V_{1}}{\partial Q_{n}} & \cdots & \frac{\partial V_{n}}{\partial Q_{n}}
\end{array}\right]=-\frac{1}{V_{n o m}}[X]}
\end{gathered}
$$

Those expressions allow quantifying voltage variations at each network node due to active and reactive power variations at any other node of a radial distribution network.

Reactance and resistance of cables are given as a value per kilometer:

$$
R_{i j}=\frac{r_{i j}}{L_{i j}} \quad \text { and } \quad X_{i j}=\frac{x_{i j}}{L_{i j}}
$$

Equation () can be expressed as:

$$
\left\{\begin{array}{l}
\left(F_{p}\right)_{i j}=\frac{\partial V_{i}}{\partial P_{j}}=-\frac{1}{V_{n o m}} L_{i j} r_{i j} \\
\left(F_{q}\right)_{i j}=\frac{\partial V_{i}}{\partial Q_{j}}=-\frac{1}{V_{n o m}} L_{i j} x_{i j}
\end{array}\right.
$$

Where $\mathrm{L}_{\mathrm{ij}}$ are:

- for $\mathrm{i}=\mathrm{j}$ the sum of the branch lengths forming the path from the origin (node 0 ) to node $\mathrm{i}$

- for $i \neq j$ the sum of the branch lengths forming the path from the origin to the common node of the paths formed by the origin and nodes $\mathrm{i}$ and $\mathrm{j}$.

A graphical representation is useful to mitigate how section and type of conductor (overhead line or underground cable line) influence the network sensitivity. And to get immediate perception of sensitivity variation with node distance from the origin.

\section{NETWORK CHARACTERISTICS AND THE IMPACT ON THE NETWORK VOLTAGE PLAN}

By considering the electrical proprieties of conductors used in [2]:

Underground cable with section $35 \mathrm{~mm}^{2}$ : $\mathrm{r}=0.675 \mathrm{Ohm} / \mathrm{km}$ and $\mathrm{x}=0.2 \mathrm{Ohm} / \mathrm{km}$

Underground cable with section $95 \mathrm{~mm}^{2}$ : $\mathrm{r}=0.249 \mathrm{Ohm} / \mathrm{km}$ and $\mathrm{x}=0.18 \mathrm{Ohm} / \mathrm{km}$

Overhead cable with section $35 \mathrm{~mm}^{2}$ : $\mathrm{r}=0.5190 \mathrm{Ohm} / \mathrm{km}$ and $\mathrm{x}=0.388 \mathrm{Ohm} / \mathrm{km}$

Overhead cable with section $95 \mathrm{~mm}^{2}$ : $\mathrm{r}=0.193 \mathrm{Ohm} / \mathrm{km}$ and $\mathrm{x}=0.357 \mathrm{Ohm} / \mathrm{km}$

Fig 6, gives a representation of the sensitivity of voltage plan with two conductors sections. Such a graphical representation is useful to get how conductors sections impact the voltage plan of the network, and it's clear that is more is section is smaller, more the influence of active and reactive on the voltage plan is more important.

Fig 7, gives a comparison between overhead and underground network, and their impact on the voltage plan of the a network with $35 \mathrm{~mm}^{2}$ conductors section, and in the fig 8 , the same comparison is done but with the $95 \mathrm{~mm}^{2}$ conductors. Fig 7 and Fig 8 shows that the undergrounded cables are more sensitive to active power variation. And the overhead lines are more sensitive to reactive power variation

For the same distance from the substation, for example $10 \mathrm{Km}$ as a distance from the substation, and in a $20 \mathrm{KV}$ network, the voltage sensitivity at a node located in $10 \mathrm{Km}$, with respect to the variations of its active and reactive power is given in table VI.

TABLE I

VOLTAGE SENSITIVITY OF A NODE LOCATED AT 10KM FROM THE SUBSTATION

\begin{tabular}{|c|c|c|r|r|}
\cline { 2 - 5 } \multicolumn{1}{c|}{} & $\begin{array}{c}\text { Cable } \\
\text { section }\end{array}$ & $\mathbf{S}_{\mathbf{p}}$ & \multicolumn{1}{c|}{$\mathbf{S}_{\mathbf{q}}$} & \multicolumn{1}{c|}{$\mathbf{S}_{\mathbf{p}} / \mathbf{S}_{\mathbf{q}}$} \\
\hline \multirow{2}{c}{$\begin{array}{c}\text { Underground } \\
\text { cable }\end{array}$} & $35 \mathrm{~mm}^{2}$ & -0.337 & -0.1 & 3.37 \\
\cline { 2 - 5 } & $95 \mathrm{~mm}^{2}$ & -0.124 & -0.09 & 1.38 \\
\hline \multirow{2}{*}{$\begin{array}{c}\text { Overhead } \\
\text { line }\end{array}$} & $35 \mathrm{~mm}^{2}$ & -0.259 & -0.194 & 1.33 \\
\cline { 2 - 5 } & $95 \mathrm{~mm}^{2}$ & -0.096 & -0.178 & 0.54 \\
\hline
\end{tabular}

Table IV shows that the undergrounded cables are more sensitive to active power variation. And the overhead lines are more sensitive to reactive power variation. We can also observe that the voltage sensitivity is more important for the $35 \mathrm{~mm}^{2}$ cables than the $95 \mathrm{~mm}^{2}$ cables. So it's to highlight that for the underground networks, the nodes voltages are more influenced by the active power variations, than the reactive power variations. And for the overhead line, the nodes voltages are more influenced by the reactive power variations, than the active power variations. And the voltage sensitivity is greater for the smaller sections.

\section{VOLTAGE SENSITIVITY AND MV DISTRIBUTION SYSTEM IN MOROCCO}

The Moroccan MV distribution networks are radial. So, to connect a new DG into a Moroccan Medium voltage distribution system, first, it's to distinguish the form of the network: $100 \%$ overhead lines, $100 \%$ underground cables or a combination of both.

For the overhead lines: it's to highlight that overhead network in Morocco had an arborescent structure, with derivations stemming from a main-line, and grouping into a cluster, as shown in fig 5 .

In such network structure, the main-line is made from conductors with bigger sections than the derivations conductors. In the case of having different possibilities to chose a connection point of a new DG, the best emplacement to connect the new DG, is the point presenting the shortest distance from the substation, and the point located in the part of the network with the biggest conductor section.

For controlling the network voltage, the most influencing regulator is a system able to adjust its consumption and injection of reactive power.

For the underground networks: The most used topology is the open loop (ring configuration) in which, each MV/LV

substation is connected to the two others substations, as shown on Fig.4. 


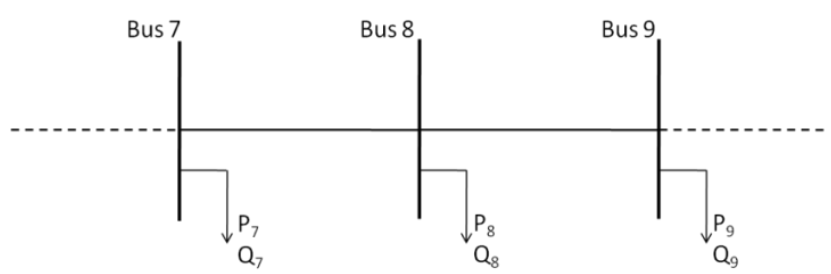

Fig 4: Single line diagram of the open-loop topology

A new DG must be connected as closer as possible to the substation, and the must influencing regulators are the systems, able to act at their consumption or injection of active powers.

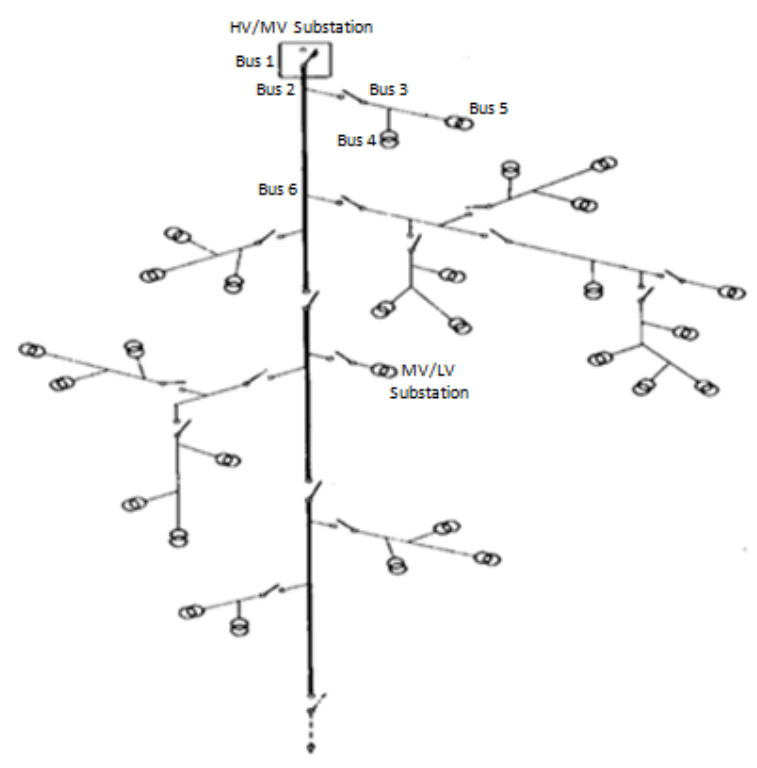

Fig 5: MV Overhead distribution system

For a network composed of overhead and underground conductor, a new DG must be connected as closer as possible to the substation, and for choosing the optimal placement of voltage regulator, it's preferable to process to the

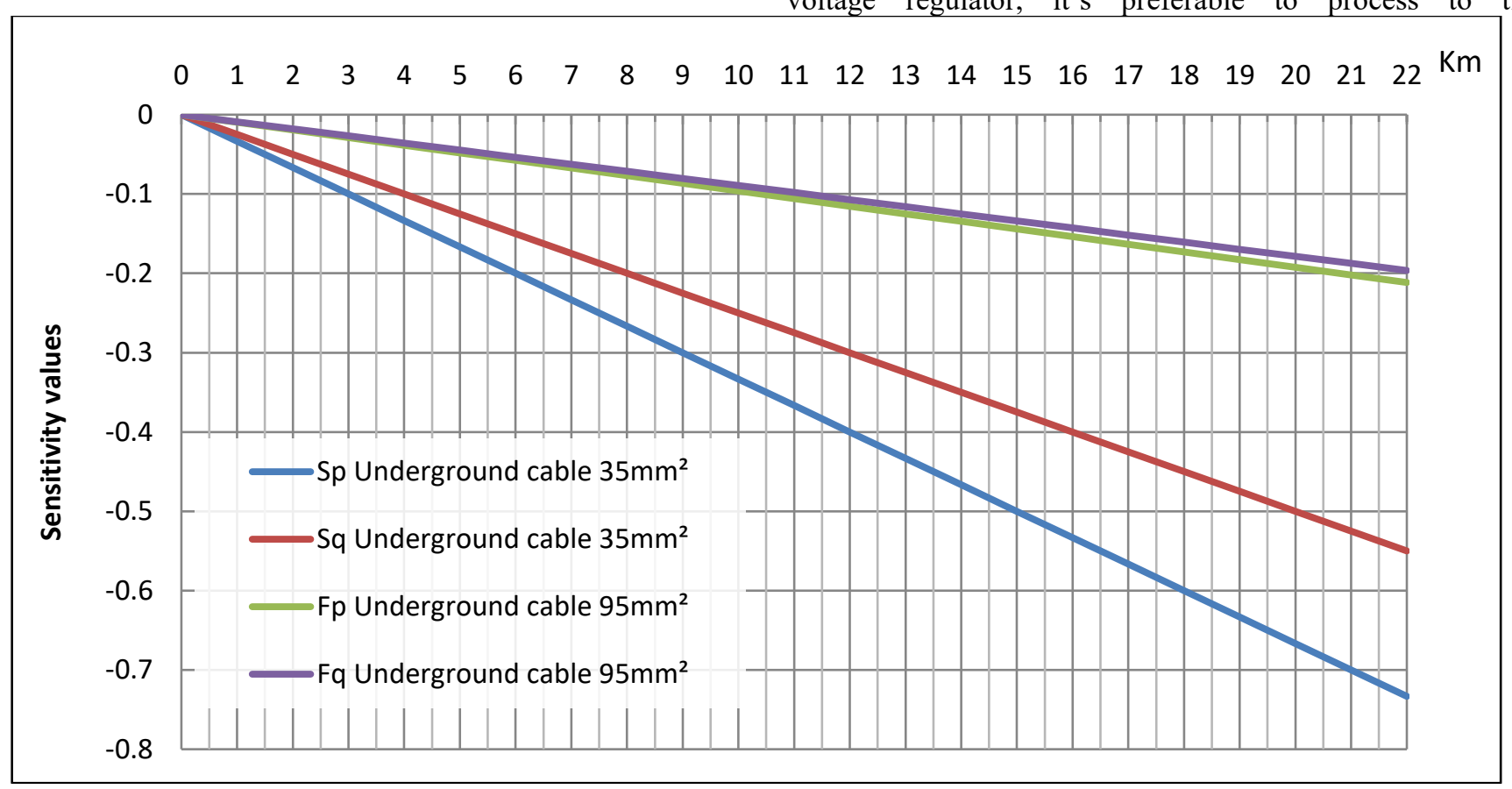

Fig 6: Graphical representation of the influence of conductor sections on voltage sensitivity. 


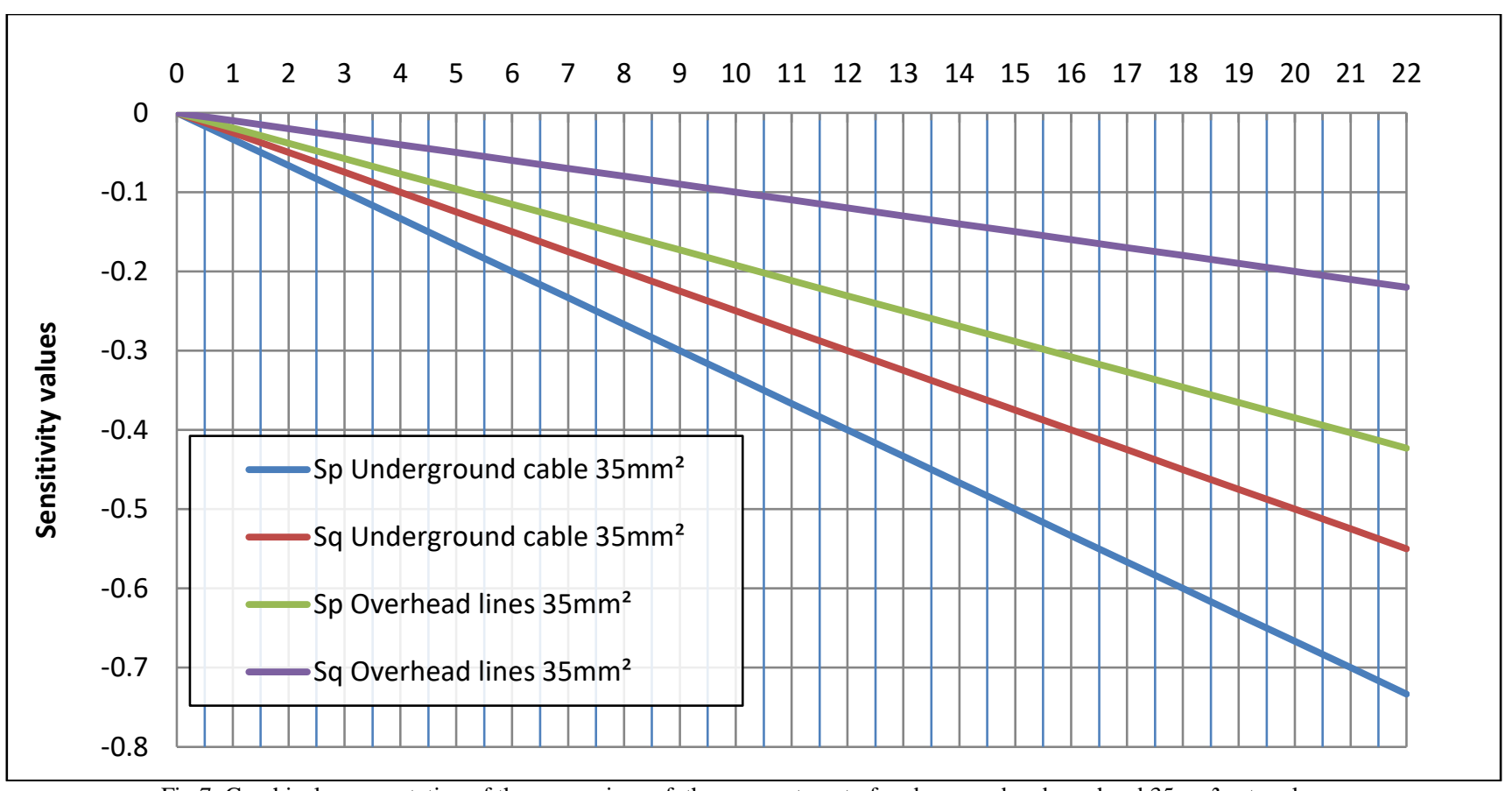

Fig 7: Graphical representation of the comparison of the comportment of underground and overhead $35 \mathrm{~mm}^{2}$ network.

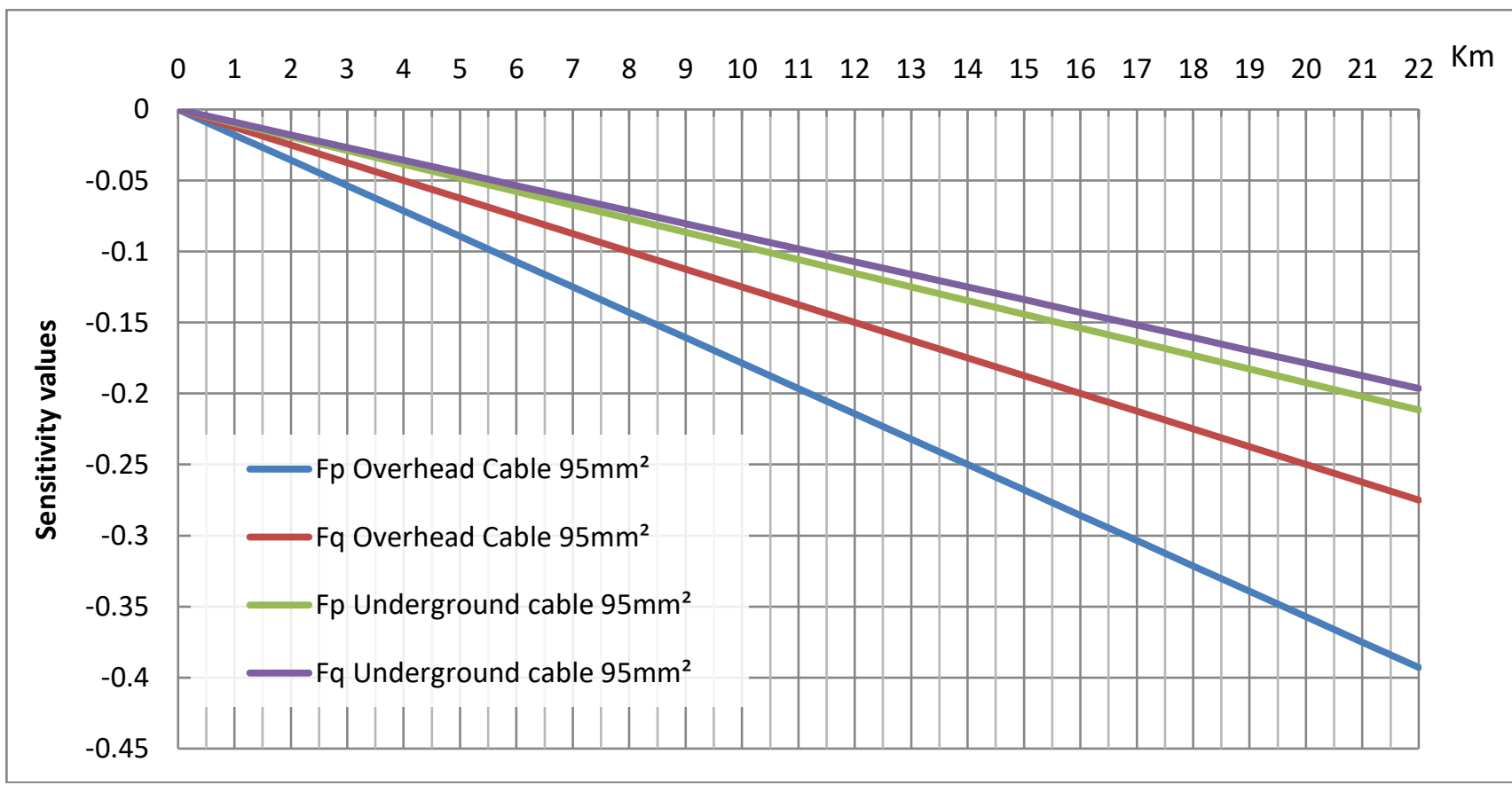

Fig 8: Graphical representation of the comparison of the comportment of underground and overhead $95 \mathrm{~mm}^{2}$ network.

computation of voltage sensitivity using the presenting method in this paper.

\section{CONCLUSIONS}

A analytical method to quantify the voltage sensitivity to the variation of active and reactive power variation is presented in this paper, The proposed method is developed based on the exploitation of the radial structure of medium voltage distribution system, and the backward/forward sweep concept, by forming two matrices: bus injection to bus current (BIBC) and branch current to bus voltage (BCBV).

As shown in this paper, the voltage of any node of the network depends on the active and reactive power of all network nodes. The proposed method is able to quantify this influence in a case of the variation of active or reactive power of any node of the network, on the voltage of any other part of the network.

The results obtained by the proposed method may be interesting for two applications:

- Choosing the connection point of a new DG, in the case of the presence of several feeders, in the vicinity of the DG site.

- Choosing the best way to control the voltage of a certain node of the network, by acting at the most influencing factor: active or reactive power and at which point. 


\section{REFERENCES}

[1] G. Mokhtari, A. Ghosh, G. Nourbakhsh, and G. Ledwich, "Smart robust resources control in $\mathrm{LV}$ network to deal with voltage rise issue," IEEE Trans. on Sustain. Energy, vol. 4, no. 4, pp. 1043-1050, Oct 2013.

[2] S.Conti, S.Raiti, G.Vagliasindi, "Voltage sensitivity analysis in radial MV distribution networks using constant current model," Industriel Electronics (ISIE), IEEE international Symposium, Nov. 2010
[3] S. OUALI, A. Cherkaoui: "Load flow analysis for moroccan medium voltage distribution system", Conférence Internationale en Automatique \& Traitement de Signal (ATS-2018), Proceedings of Engineering and Technology - PET, Vol.36 pp.10-16

[4] G.W Stagg and A.H. El-Abiad, Computer Methods in Power System Analysis, McGraw Hill, 1968. 